\title{
Treatment of Dysphagia with Pyridostigmine Bromide in a Patient with the Pharyngeal- Cervical-Brachial Variant of Guillain-Barré Syndrome
}

Kwang Lae Lee, M.D., Oh Kyung Lim, M.D., Ph.D. ${ }^{1}$, Ju Kang Lee, M.D., Ph.D. ${ }^{1}$, Ki Deok Park, M.D.

Department of Rehabilitation Medicine, National Rehabilitation Hospital, Seoul 142-884,

${ }^{1}$ Gachon University of Medicine and Science, Incheon 405-760, Korea

\begin{abstract}
A 24-year-old male developed bulbar palsy, ophthalmoplegia, ptosis, and shoulder weakness bilaterally 2 weeks after he had experienced an upper respiratory infection. The electrodiagnostic study demonstrated axonal polyradiculoneuropathy. The repetitive nerve stimulation study (RNS) showed no significant decrement of the compound muscle action potentials (CMAPs). The videofluoroscopic swallowing study (VFSS) showed severe impairment of the pharyngeal phase of swallowing. He was diagnosed as having the pharyngeal-cervical-brachial variant of Guillain-Barré syndrome. The patient's dysphagia was not improved for 3 months. A follow up RNS showed a significant decrement of the CMAPs. Pyridostigmine bromide was tried to improve the dysphagia. The patient showed immediate improvement of his dysphagia on the VFSS after the trial with pyridostigmine bromide. Pyridostigmine bromide was given before each meal for 8 days and he showed continuous improvement of his dysphagia. The follow up VFSS after 3 months showed complete recovery of dysphagia.
\end{abstract}

Key Words Guillain-Barré syndrome, Dysphagia, Pyridostigmine bromide

\section{INTRODUCTION}

The pharyngeal-cervical-brachial (PCB) variant of Guillain-Barré syndrome (GBS) is very rare and is characterized by weakness of the pharyngeal, cervical, and brachi-

Received September 2, 2010; Accepted April 15, 2011

Corresponding author: Ju Kang Lee

Department of Rehabilitation Medicine, Gachon University of Medicine and Science, 1198, Guwol-dong, Namdong-gu, Incheon 405-760, Korea

Tel: +82-32-460-3722, Fax: +82-32-460-3722, E-mail: pm@gilhospital.com () This is an open-access article distributed under the terms of the Creative Commons Attribution Non-Commercial License (http:// creativecommons.org/licenses/by-nc/3.0) which permits unrestricted noncommercial use, distribution, and reproduction in any medium, provided the original work is properly cited.

Copyright $\odot 2012$ by Korean Academy of Rehabilitation Medicine al muscles. ${ }^{1}$ The prognosis of this variant generally shows a slow and incomplete recovery over several months. ${ }^{2,3}$ It has been reported that the ptosis associated with the PCB variant is slightly improved by administration of neostigmine or edrophonium, but the other symptoms are not improved. ${ }^{3}$ We treated severe dysphagia in a patient suffering with the PCB variant by administering pyridostigmine bromide. Here, we report on our case and provide a review of the relevant literature.

\section{CASE REPORT}

A 24-year-old male patient developed whole body myalgia, ptosis and neck muscle weakness (day 1) after ex- 
periencing 2 weeks of cough, rhinorrhea, mild fever, and sore throat. He took cold medicine, but developed dysphagia, dysarthria, and shoulder weakness bilaterally 4 days later. On day 4, he was admitted to the Department of Neurology at the University Hospital.

At the time of admission, he had ptosis, dysarthria, diplopia, dysphagia, facial palsy, ophthalmoplegia, and ataxia without hemisensory disturbance. His consciousness was not impaired. Manual muscle testing revealed a $-3 / 5$ weakness in the cervical and shoulder muscles and a $-4 / 5$ weakness in the other muscles. The sensory testing and the deep tendon reflexes showed normal findings. There were no signs of meningeal irritation, and the brain magnetic resonance imaging (MRI) did not show any abnormal lesion. Laboratory test results such as antiacetylcholine receptor antibodies, creatine kinase, aspartate aminotransferase, alanine aminotransferase, lactate dehydrogenase, and C-reactive protein were normal. The cerebrospinal fluid study showed no signs of infection or albuminocytologic dissociation. No abnormalities of the thymus were shown on computed tomography. An electrodiagnostic study was performed on day 38 . The repetitive nerve stimulation study (RNS) did not show a significant decrement of the compound muscle action potentials (CMAPs) in the abductor digiti minimi and orbicularis oculi muscles. The nerve conduction and $\mathrm{F}$ wave studies showed normal findings, but there were abnormal spontaneous activities at the muscles of all the extremities and this was compatible with the axonal polyradiculoneuropathy seen on the needle electromyography.

Based on the symptoms and examinations, the patient was diagnosed as having GBS, and we started treatment with immunoglobulin. He was given 25,000 mg immunoglobulin intravenously daily for 4 days. The ptosis, diplopia, facial palsy, ophthalmoplegia, limb muscle weakness, and ataxia were improved after immunoglobulin therapy and physical therapy, but the dysphagia was not improved. The videofluoroscopic swallowing study (VFSS) on day 40 showed severe dysfunctional oropharyngeal transfer of the barium paste and subglottic aspiration of the liquid barium due to the severe weakness and lack of coordination of the oropharyngeal muscles. Oromotor facilitation and electrical stimulation therapy to the pharyngeal muscles was done for the swallowing difficulty. The waveform, amplitude, duration, and fre-

Table 1. Results of Sensory Nerve Conduction Studies

\begin{tabular}{llccc}
\hline \multicolumn{1}{c}{ Nerve } & Stimulation & Recording & DL (msec) & Amplitude* $(\boldsymbol{\mu V})$ \\
\hline Median & Wrist & Second digit & 2.80 & 80.8 \\
Ulnar & Wrist & Fifth digit & 2.00 & 53.0 \\
Superficial peroneal & Lateral leg & Foot & 2.30 & 21.3 \\
Sural & Calf & Lateral malleolus & 2.30 & 21.3 \\
\hline
\end{tabular}

DL: Distal latency (onset latency)

*Baseline to peak amplitude

Table 2. Results of Motor Nerve Conduction Studies

\begin{tabular}{|c|c|c|c|c|c|}
\hline Nerve & Stimulation & Recording & DL (msec) & Amplitude* (mV) & Velocity $^{\dagger}(\mathrm{m} / \mathrm{s})$ \\
\hline \multirow[t]{2}{*}{ Median } & Wrist & APB & 3.70 & 6.9 & \\
\hline & Elbow & & & 6.8 & 57.1 \\
\hline \multirow[t]{2}{*}{ Ulnar } & Wrist & $\mathrm{ADM}$ & 2.65 & 6.0 & \\
\hline & Elbow & & & 5.9 & 55.3 \\
\hline \multirow[t]{2}{*}{ Common peroneal } & Ankle & EDB & 3.85 & 2.3 & \\
\hline & Fibular head & & & 2.3 & 50.4 \\
\hline \multirow[t]{2}{*}{ Tibial } & Ankle & $\mathrm{AH}$ & 3.55 & 13.3 & \\
\hline & Knee & & & 10.6 & 53.4 \\
\hline
\end{tabular}

APB: Abductor pollicis brevis, ADM: Abductor digit minimi, EDB: Extensor digitorum brevis, AH: Abductor hallucis, DL: Distal latency (onset latency)

${ }^{*}$ Baseline to peak amplitude, ${ }^{\dagger}$ Segmental nerve conduction velocity from the proximal site to the distal site 
Table 3. Results of Needle Electromyography

\begin{tabular}{lll}
\hline Muscle (Right) & \multicolumn{1}{c}{ ASA } & \multicolumn{1}{c}{ MUAP } \\
\hline APB/ADM/FDI & Fib/PSW $(+++)$ & Long duration/polyphasic \\
\hline FCR/FCU & Fib/PSW $(+++)$ & Long duration/polyphasic \\
\hline Biceps brachii & Fib/PSW $(+++)$ & Long duration/polyphasic \\
Triceps & Fib/PSW $(+++)$ & Long duration/polyphasic \\
Deltoid & Fib/PSW $(+++)$ & Long duration/polyphasic \\
Cervical paraspinal & Fib/PSW $(+++)$ & Long duration/polyphasic \\
Tibialis anterior & Fib/PSW $(+++)$ & Long duration/polyphasic \\
Peroneus longus & Fib/PSW $(+++)$ & Long duration/polyphasic \\
Gastrocnemius & Fib/PSW $(+++)$ & Long duration/polyphasic \\
Vastus medialis & Fib/PSW $(+++)$ & Long duration/polyphasic \\
TFL & Fib/PSW $(+++)$ & Long duration/polyphasic \\
Lumbar paraspinal & Fib/PSW $(+++)$ & Long duration/polyphasic \\
\hline
\end{tabular}

ASA: Abnormal spontaneous activities, MUAP: Motor unit action potentials, APB: Abductor pollicis brevis, ADM: Abductor digit minimi, FDI: First dorsal interosseous, FCR: Flexor carpi radialis, FCU: Flexor carpi ulnaris, TFL: Tensor fascia lata, Fib: Fibrillation potentials, PSW: Positive sharp waves

quency of the electrical stimulator were rectangular symmetric biphasic, $7 \mathrm{~mA}, 300 \mathrm{msec}$, and $80 \mathrm{~Hz}$, respectively. The follow up VFSS on day 54 did not show improvement of the dysphagia.

He was transferred to the Department of Rehabilitation Medicine for treatment of the dysphagia on day 61. At the time of transfer, he was being maintained on nasogastric tube feeding and complained of mild dysarthria. The tongue muscles showed weakness and the soft palate movement was impaired. He had mild facial weakness. Manual muscle testing revealed 4/5 weakness in the shoulder and hip muscles bilaterally with normal muscle power in the other muscles. The signs and symptoms were persistent and they could not be improved by rest. Antibodies for CMV, EBV, and anti-GM1 IgG antibodies were detected in the serum on day 70 . Anti-acetylcholine receptor antibodies were not detected. The weakness of the shoulder and hip muscles was improved after intensive rehabilitation, but the dysphagia persisted. A follow up electrodiagnostic study was performed on day 70 . The nerve conduction study on the right limbs showed normal sensory fiber conductions and normal motor fiber conduction velocities, but there was decreased amplitude of the CMAPs (Table 1 and 2). The F wave studies at the median, ulnar, common peroneal and tibial nerves showed normal findings. Needle electromyography showed abnormal spontaneous activities at the muscles of all the extremities and some evidence of partial axonal
Table 4. Results of Repetitive Nerve Stimulation Study on the Right Orbicularis Oculi Muscle

\begin{tabular}{lcc}
\hline \multicolumn{1}{c}{ Time } & $\begin{array}{c}\text { Amplitude* } \\
\text { (mV) }\end{array}$ & $\begin{array}{c}\text { Percentage }^{\dagger} \\
\text { (\%) }\end{array}$ \\
\hline Before exercise & 0.5 & 5.8 \\
Immediately after exercise & 0.9 & 4.9 \\
1 minute after exercise & 0.9 & 11.6 \\
10 minutes after exercise & 0.6 & 4.7 \\
\hline
\end{tabular}

*Amplitudes of the first compound muscle action potentials, ${ }^{\dagger}$ The percentages of amplitude decrements of the fifth compound muscle action potentials as compared to the first ones

regeneration as compared to the previous study (Table 3). RNS showed a significant decrement of the CMAPs at the orbicularis oculi muscle on $3 \mathrm{~Hz}$ repetitive simulation (Table 4). We interpreted that these findings of dysfunction of the conduction at the neuromuscular junction were caused by the axonal polyradiculoneuropathy, and decided to perform a pyridostigmine challenge test for the dysphagia to confirm this interpretation.

The pyridostigmine challenge test was performed with VFSS on day 75. Before administering the pyridostigmine, fluoroscopic monitoring revealed pharyngeal retention and aspiration of barium coated food without any passage to the esophagus, which suggested no improvement of the pharyngeal weakness. However, 30 minutes after oral administration of pyridostigmine $(60 \mathrm{mg})$, the 

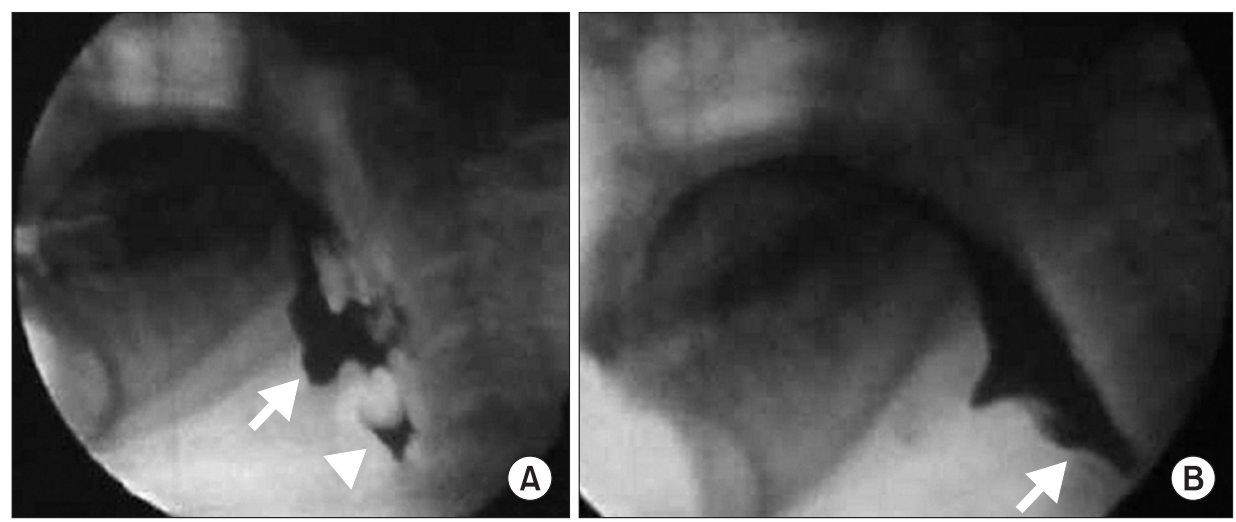

Fig. 1. (A) Barium liquid did not transfer to the esophageal phase because of pharyngeal muscle weakness. The image showed severe retention of material at the vallecula (white arrow) and the pyriform sinus (triangle). (B) The same material transferred to the esophageal phase 30 minutes after taking pyridostigmine bromide.

amount of retention significantly decreased and food passage to the esophagus was clearly seen (Fig. 1). With this finding we confirm the effect of pyridostigmine for the treatment of dysphagia in this case. Since this study, pyridostigmine bromide (60 $\mathrm{mg}$ ) was regularly given to the patient 30 minutes before each meal time, and the patient could swallow a soft diet with some difficulty. Within several days of pyridostigmine administration, the swallowing difficulty was significantly improved and the nasogastric tube was removed. On day 83, the patient could eat a full oral diet without pyridostigmine. On day 89, the follow up VFSS showed further improvement of the pharyngeal weakness and no aspiration of any material.

He was discharged on day 90. The follow up VFSS on day 173 showed full recovery from the pharyngeal weakness and a normal swallowing function.

\section{DISCUSSION}

The PCB variant of GBS is diagnosed if the following conditions are satisfied; progressive severe weakness of the pharyngeal, cervical, and brachial muscles, and the absence or decrement of the deep tendon reflexes of the arm in a week and 3 weeks, respectively, mild weakness of the lower extremities $>$ grade $4 / 5$, a past history of infection in the 4 weeks before the neurologic presentation, albuminocytologic dissociation on the cerebrospinal fluid study, and electrophysiological abnormalities of the conduction velocities. ${ }^{4}$ However, it is possible that normal or increased deep tendon reflexes can be seen and the cerebrospinal study is not able to show albuminocytologic dissociation. It has been reported that electromyography reveals only decrements of amplitudes on the CMAPs or even normal findings. Therefore, it is proper that a patient with GBS is diagnosed to have the PCB variant if he does not show a typical presentation, but rather the patient having the PCB variant shows unusual presentations such as weakness of the pharyngeal, cervical, and brachial muscles. ${ }^{2,3}$

The PCB variant must be distinguished from botulism, diphtheria infection, myasthenia gravis, and multiple sclerosis. ${ }^{2,3}$ In our patient, we could exclude botulism and diphtheria infection according to the clinical findings and electrophysiological findings and there was no evidence of infection. We could rule out multiple sclerosis by the cerebrospinal fluid study, MRI, and electromyography. The RNS done on day 70 showed a significant decrement of the CMAPs, but there were abnormal spontaneous activities at all the muscles we examined and there was no evidence of fatigue by exercise. The signs and symptoms of our patient were persistent and could not be improved by rest. Anti-acetylcholine receptor antibodies were not detected. An acetylcholine esterase inhibitor can improve symptoms, but it does not affect the prognosis. ${ }^{5}$ The patient took the medicine for only 8 days. If the disease was myasthenia gravis, the dysphagia would recur after the cessation of the medicine. The RNS showed no decrement at the time of the symptoms of diplopia, ptosis, facial palsy, and ophthalmoplegia. On the basis of the above findings, we could rule out myasthenia gravis. The decrement of CMAPs on the RNS can be explained by the exocytosis of insufficient acetylcholine at the immature nerve endings in the course of recovery from the peripheral neuropathy with axonal damage. ${ }^{6}$ It is speculated that in our patient, the early RNS showed normal findings, but later the RNS showed a significant decrement by dysfunction of the conduction at the neuromuscular junction developing during the course of recovery from the axonal peripheral neuropathy. We could diagnose 
this case as the PCB variant of GBS according to the clinical course and the laboratory findings.

GBS is a type of autoimmune disease and it is usually preceded by upper respiratory or gastrointestinal infection. On the basis of a recent study, infections by Campylobacter jejuni, CMV, EBV and Mycoplasma pneumoniae appear to be the causes of GBS. ${ }^{7}$ A definite mechanism has not been established, but it has been revealed that the antibodies generated by the activation of immune responses after these infections make a cross-bridge via molecular mimicry with antigens in the peripheral nerves and this destroys them. ${ }^{7,8}$ This hypothesis is supported by the fact that anti-ganglioside antibodies have been found in the serum of GBS patients. These antibodies assist in making the diagnosis. ${ }^{7}$ In the PCB variant, serum antiGTla IgG antibody is usually revealed because a GT1a ganglioside is abundantly detected at the cranial nerves such as the glossopharyngeal and vagus nerves. But other antibodies such as serum anti-GQ1b and anti-GM1 IgG are occasionally revealed. ${ }^{9}$ Our patient showed 2 types of viral antibodies. This result is thought to indicate that there was a co-infection or one infection after the other infection during his hospitalization. Yet this examination had a limitation as it checked only a IgG without IgM. There was definitely a viral infection in his recent past history and we can speculate that the formation of serum anti-GM1 IgG antibody affected the occurrence of disease.

Pyridostigmine bromide is an acetylcholine esterase inhibitor used to treat myasthenia gravis. This medication delays the elimination of acetylcholine in the neuromuscular junction by inhibiting acetylcholine esterase and therefore activates neuromuscular transmission. ${ }^{5}$ On the hypothesis that this medication could improve the dysphagia of our patient, who showed a significant decrement of CMAPs on the RNS, we tried using it to treat his symptoms. His dysphagia had not been improved by $>2$ months of occupational therapy. However, on day 75 , the VFSS performed after taking the medication showed improvement of the dysphagia. We could rule out spontaneous recovery of dysphagia because the result was different from that at 30 minutes before taking the medication. Although we don't know whether only taking the medication changed the prognosis of the disease, we thought that the medication helped to improve the dysphagia and the patient, who previously could not swallow, became able to swallow. Swallowing training is generally the best therapy for swallowing disorders. ${ }^{10}$ In conclusion, we think the medication helped the pharyngeal muscle to strongly contract due to the effect on the delay of the elimination of acetylcholine in the neuromuscular junction and the more active dysphagia training through swallowing could have changed the prognosis of the dysphagia. The limitation of our study is that follow up RNS should have been done after remission of the dysphagia to test whether the medication affected transmission at the neuromuscular junction and therefore improved the dysphagia, but this was not performed. We cannot completely rule out the possibility of a spontaneous recovery.

Our case indicates that dysphagia rehabilitation by administering acetylcholine esterase inhibitor is possibly a sound method for treating the dysphagia in those GBS patients with a significant decrement of the CMAPs seen on a RNS. A study with a large population focusing on the mechanism of improvement and the possibility of using this treatment is needed in the future.

\section{REFERENCES}

1. Ropper AH. Unusual clinical variants and signs in Guillain-Barre syndrome. Arch Neurol 1986; 43: 11501152

2. Arai M, Susuki K, Koga M. Axonal pharyngeal-cervical-brachial variant of Guillain-Barre syndrome without Anti-GT1a IgG antibody. Muscle Nerve 2003; 28: 246-250

3. Kim SY, Kim SM, Yu KH, Kwon KH, Lee BC. Pharyngeal-cervical-brachial variants of Guillain-Barre syndrome. J Korean Neurol Assoc 1994; 12: 164-169

4. Asbury AK, Cornblath DR. Assessment of current diagnostic criteria for Guillain-Barre syndrome. Ann Neurol 1990; 27 Suppl: S21-24

5. Thomann KH, Pandya S. Myasthenia gravis: pathophysiology, diagnosis, differential diagnosis and management. Clinical Eye and Vision Care 1995; 7: 3-13

6. Miglietta OE. Myasthenic-like response in patients with neuropathy. Am J Phys Med 1971; 50: 1-16

7. Hughes RA, Hadden RD, Gregson NA, Smith KJ. Pathogenesis of Guillain-Barre syndrome. J Neuroimmunol 1999; 100: 74-97

8. Mogale KD, Antony JH, Ryan MM. The pharyngealcervical-brachial form of Guillain-Barre syndrome in 
childhood. Pediatr Neurol 2005; 33: 285-288

9. Murakami N, Tomita Y, Koga M, Takahashi E, Katada Y, Sakuta R, Nagai T. An adolescent with pharyngealcervical-brachial variant of Guillain-Barre syndrome after cytomegalovirus infection. Brain Dev 2006; 28:
269-271

10. Palmer JB, Drennan JC, Baba M. Evaluation and treatment of swallowing impairments. Am Fam Physician 2000; 61: 2453-2462 